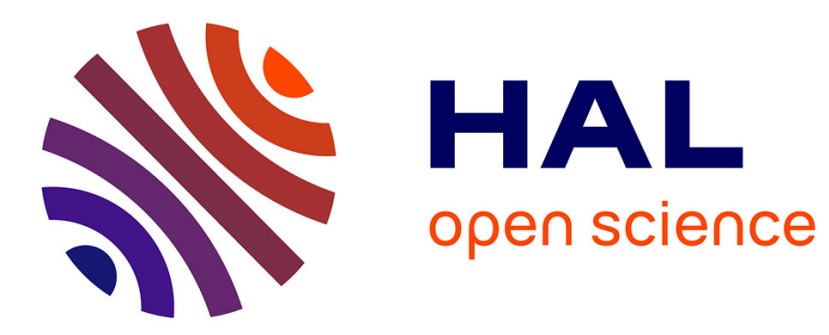

\title{
Mesure du courant d'injection de charge dans les diélectriques solides sous tension alternative
}

\author{
Thierry Lebey, C. Laurent
}

\section{To cite this version:}

Thierry Lebey, C. Laurent. Mesure du courant d'injection de charge dans les diélectriques solides sous tension alternative. Revue de Physique Appliquée, 1990, 25 (5), pp.423-433. 10.1051/rphysap:01990002505042300 . jpa-00246201

\section{HAL Id: jpa-00246201 https://hal.science/jpa-00246201}

Submitted on 1 Jan 1990

HAL is a multi-disciplinary open access archive for the deposit and dissemination of scientific research documents, whether they are published or not. The documents may come from teaching and research institutions in France or abroad, or from public or private research centers.
L'archive ouverte pluridisciplinaire HAL, est destinée au dépôt et à la diffusion de documents scientifiques de niveau recherche, publiés ou non, émanant des établissements d'enseignement et de recherche français ou étrangers, des laboratoires publics ou privés. 


\title{
Mesure du courant d'injection de charge dans les diélectriques solides sous tension alternative
}

\author{
T. Lebey et C. Laurent \\ Laboratoire de Génie Electrique, CNRS UA 304, Université Paul Sabatier, 118 Route de Narbonne, 31062 \\ Toulouse Cedex, France
}

(Reçu le 7 décembre 1989, accepté le 5 fëvrier 1990)

\begin{abstract}
Résumé. - On mesure le courant d'injection de charge créé par concentration locale du champ électrique à l'extrémité d'une pointe moulée dans un isolant polymère soumis à une tension sinusoïdale. Le détecteur permet la mesure analogique de la charge injectée à tout instant de la contrainte avec une sensibilité de l'ordre de $10^{-14} \mathrm{C}$. Dans les matériaux étudiés (polyéthylène et polyépoxy), l'injection n'est effective qu'au delà du champ critique $(\approx 1 \mathrm{MV} / \mathrm{cm})$. Le courant change de signe au cours de chaque alternance (injection/extraction de charges) et l'injection est ambipolaire. Le diélectrique acquiert une charge permanente après un temps de polarisation suffisamment long. L'importance de la mesure du courant d'injection dans le domaine du vieillissement électrique des isolants est discutée.
\end{abstract}

\begin{abstract}
The charge flow injected during a sinusoidal excitation from a needle electrode into dielectrics is measured. The experimental set up allows the analog, time resolved measurement of the charge down to a sensitivity of $10^{-14} \mathrm{C}$. In polyethylene and epoxy samples, the injection sets in at a precise field level called the critical field $(\simeq 1 \mathrm{MV} / \mathrm{cm}$ ). Charge is injected and extracted during each cycle, and the injection is ambipolar. A permanent charge appears in the dielectric after a long stressing period. The injected space charge measurement seems to be of importance regarding the electrical ageing of dielectrics.
\end{abstract}

\section{Introduction.}

Un diélectrique est généralement considéré comme démuni d'électrons libres. Sous l'action d'un champ électrique, des électrons provenant du métal des électrodes pénètrent dans le matériau et en modifient la conductivité : c'est le mécanisme d'injection. Deux régimes de conduction peuvent alors être définis suivant la valeur de la tension appliquée à l'isolant. Pour de faibles tensions, les porteurs injectés se déplacent dans le diélectrique et le traversent en un temps long comparé au temps de relaxation du matériau. La mobilité reste la mobilité intrinsèque, et la conduction peut être considérée comme ohmique. Pour des tensions plus élevées, le temps de transit des porteurs injectés devient très court par rapport au temps de relaxation et cette charge détermine la distribution du champ électrique dans le volume du diélectrique. La mobilité peut alors être considérée dans la zone de charge d'espace, comme une mobilité de bande. Dans le cas du polyéthylène, polymère très largement utilisé comme isolant haute tension, la mobilité intrinsèque est de l'ordre de $10^{-10}$ à $10^{-8} \mathrm{~cm}^{2} / V . s$. tandis que la mobilité de bande atteindrait des valeurs de l'ordre de 1 à $10 \mathrm{~cm}^{2} /$ V.s. [1]. L'importance des phénomènes d'injection sur la tenue diélectrique du matériau est bien connue. D'une part, les porteurs injectés modifient la répartition du champ électrique au sein des échantillons. L'existence d'une charge d'espace au voisinage des électrodes permet par exemple d'expliquer les différences constatées expérimentalement entre rigidité diélectrique sous contrainte impulsionnelle et continue, et les effets de polarité. La structure du diélectrique peut, d'autre part, être affectée par des réactions de dissociation consécutives à l'impact d'électrons chauds sur des molécules, ou à l'absorption de l'énergie relaxée par le piégeage ou la recombinaison des porteurs. Ces derniers phénomènes sont particulièrement importants sous tension alternative puisque la nature des porteurs injectés dépend du signe de l'alternance. Enfin, il semble aujourd'hui bien établi que le vieillissement 
électrique des matériaux en alternatif est étroitement lié à l'injection de charges [2]. On comprend ainsi tout l'intérêt que revêt sa détection dans les diélectriques sous tension alternative. Les techniques de mesure de la charge injectée dans les diélectriques sont nombreuses. Certaines permettent une caractérisation de la charge résiduelle après polarisation. Citons par exemple les mesures de courant de dépolarisation stimulée [3], de courant induit par ondes acoustiques [4] ou thermiques [5]. Les informations qu'elles donnent sont cependant peu intéressantes dans le cas d'une contrainte alternative puisque la charge persistante mesurée après polarisation n'a que peu de rapport avec celle réellement mise en jeu. Récemment, des techniques de mesure de la charge injectée sous contrainte ont été mises au point. Ces dispositifs sont décrits dans la littérature. Rappelons que deux systèmes sont essentiellement utilisés. Le pont de Lemke [6] permet la mesure de la charge cumulée mise en jeu dans une configuration pointe plan jusqu'à l'apparition d'une arborescence électrique. Ce dispositif est cependant peu sensible $(\simeq 1 \mathrm{pC})$ et ne permet pas la mesure du courant d'injection en temps réel. Le système mis au point par Hibma et al. [7] est beaucoup plus sensible $\left(\approx 10^{-3} \mathrm{pC}\right)$ et permet un suivi temporel de la charge injectée à l'extrémité d'une électrode pointe. La compensation numérique des composantes linéaires du courant attribuées aux effets de relaxation présente cependant quelques inconvénients lors du test d'échantillons spécifiques [8]. Le système que nous proposons évite certains de ces inconvénients et trouve son originalité dans le fait qu'il permet une mesure entièrement analogique du courant d'injection.

\section{Principe de la détection.}

2.1 CONCEPT. - L'idée centrale sur laquelle repose le principe de notre système de détection est l'existence probable d'un changement abrupt de la mobilité des porteurs injectés à partir d'une certaine valeur du champ, dit champ critique $E_{\mathrm{c}}$. Ce concept a été développé par Zeller dans le cadre de la théorie FLSC [7]. Dans la pratique, c'est une tension qui est appliquée au matériau, et non un champ. Le champ critique d'injection $E_{\mathrm{c}}$ est alors relié à la tension critique $V_{\mathrm{c}}$ par la géométrie du site à partir duquel l'injection se produit.

2.2 PRINCIPE DE LA DÉTECTION. - Dans l'hypothèse de l'existence d'une tension critique pour l'injection telle que nous venons de la définir, considérons une diélectrique placé entre deux armatures métalliques et inscrit dans un circuit électrique en série avec une source de tension sinusoïdale. Tant que la tension appliquée $V$ reste inférieure à la tension critique $V_{\mathrm{c}}$, la charge totale aux bornes de l'échantillon peut se décomposer en deux termes:

- une charge capacitive $Q_{\mathrm{c}}$ égale au produit de la capacité de l'échantillon par la tension appliquée,

- une charge de polarisation $Q_{\mathrm{p}}$ liée à la dépendance fréquentielle de la constante diélectrique $\varepsilon_{\mathrm{r}}(\omega)$,

soit :

$$
Q_{\mathrm{t}}=Q_{\mathrm{c}}+Q_{\mathrm{p}}
$$

Quand $V$ excède $V_{\mathrm{c}}$, un troisième terme lié à l'injection de charges dans le matériau doit être pris en compte. Soit $Q_{\text {ce }}$ cette charge. On a :

$$
Q_{\mathrm{t}}=Q_{\mathrm{c}}+Q_{\mathrm{p}}+Q_{\mathrm{ce}}
$$

$Q_{\mathrm{ce}}$ est la contribution de la charge d'espace à la charge totale, englobant donc aussi les charges images induites sur l'électrode injectante.

La détermination de la quantité $Q_{\mathrm{ce}}$ est extrêmement difficile puisque la charge capacitive lui est supérieure de plusieurs ordres de grandeur. Afin d'atténuer cette difficulté, nous avons choisi une configuration d'électrodes de type double pointe dans laquelle la capacité est réduite à $\simeq 10^{-13} \mathrm{~F}$, contre $10^{-11} \mathrm{~F}$ dans une configuration pointe plan. Dans ces conditions, le rapport $Q_{\mathrm{c}} / Q_{\mathrm{ce}} \simeq 10^{+5}$ à $1 \mathrm{kV}$. Le principe de la mesure repose alors sur les hypothèses suivantes :

- les charges capacitives $Q_{\mathrm{c}}$ et de polarisation $Q_{\mathrm{p}}$ peuvent être compensées avec suffisamment de précision par des charges de même nature ;

- la charge de polarisation est linéaire avec la tension appliquée.

Le schéma décrivant les diverses opérations réalisées par notre dispositif expérimental est donné à la figure 1. Nous utilisons deux échantillons préparés dans des conditions strictement identiques et soumis à des tensions différentes $V_{1}$ et $V_{2}=m V_{1}$, avec $m>1$. Au cours d'une première étape, les charges capacitives $Q_{\mathrm{c} 1}$ et $Q_{\mathrm{c} 2}$ apparaissant aux bornes de chacun des échantillons sont compensées par sommation de deux autres charges capacitives $-Q_{\mathrm{cl}}$ et - $Q_{\mathrm{c} 2}$. Au cours d'une seconde étape, on procède à la multiplication analogique par $m$ de la charge de polarisation $Q_{\mathrm{pl}}$. La dernière étape est une soustraction des signaux obtenus. Elle conduit théoriquement à :

avec

$$
\begin{aligned}
-Q_{\mathrm{t}} & =0 \text { quand } V<V_{\mathrm{c}} \\
-Q_{\mathrm{t}} & =Q_{\mathrm{ce}} \text { quand } V>V_{\mathrm{c}} \\
V & =V_{2} .
\end{aligned}
$$

\section{Echantillons et dispositif expérimental.}

3.1 EChANTILlONS. - Deux isolants polymères très utilisés en électrotechnique sont étudiés. Il s'agit d'un polyéthylène basse densité et d'un polyépoxy. 


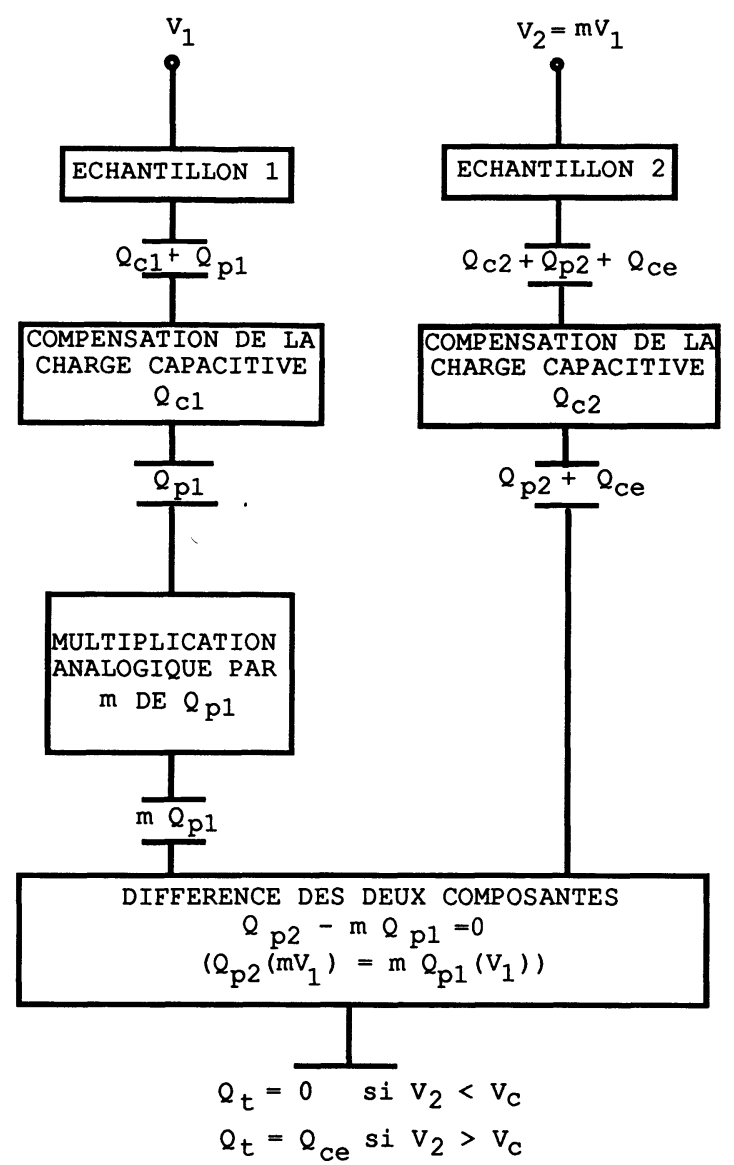

Fig. 1. - Schéma de principe du détecteur. Les différentes opérations sont effectuées à faible niveau pour vérifier $V_{2}<V_{\mathrm{c}}$. Le détecteur est dit équilibré lorsque la charge de sortie est nulle. Comme nous le verrons dans la pratique, $Q_{\mathrm{t}}$ n'est jamais nulle mais peut être minimisée.

[Principle of the charge detector. The operations at the different steps are realized under a low imput voltage in order to fulfill the condition $V_{2}<V_{\mathrm{c}}$. The set up is said to be tuned when the output charge $Q_{\mathrm{t}}$ is zero. In practice, $Q_{\mathrm{t}}$ is never zero but can be minimized.]

La configuration des électrodes est du type double pointe assymétrique (Fig. 2). Une des électrodes sert de site d'amplification du champ électrique. Elle présente un rayon de courbure en pointe $r$ variable de 3 à $10 \mu \mathrm{m}$ selon les expériences. L'autre électrode coaxiale a un rayon de courbure constant, $R$, égal à $500 \mu \mathrm{m}$. Ces électrodes en acier inoxydable sont fournies par la Société Ogura Jewels Limited et présentent des caractéristiques géométriques reproductibles. La valeur du rayon de courbure de chaque électrode est toutefois contrôlée par une mesure directe effectuée sous microscope. Compte-tenu des notations utilisées à la figure 2 , le champ électrique maximum en pointe d'électrode est donnée par la formule [9] :

$$
E_{\max }=\frac{2 V}{r \log \left(2 d\left(1+\frac{2 d}{R}\right) / r\right)} .
$$

L'utilisation d'une configuration double pointe nous permet, d'une part d'obtenir des champs importants en pointe à partir de valeurs relativement faibles de la tension appliquée, et d'autre part, comme nous l'avons déjà souligné, de minimiser la charge capacitive de nos échantillons.

Ces derniers sont préparés : lène

- par polymérisation de la résine pour le polyépoxy.

Les difficultés majeures rencontrées au cours de la préparation des éprouvettes sont liées aux coefficients de dilatation thermique différents entre métal et polymère. Un traitement préalable des électrodes permet cependant la réalisation d'échantillons de bonne qualité ne présentant pas de décollement.

\subsection{LE DÉTECTEUR DE CHARGE.}

3.2.1 Montage électronique. - L'ensemble des opérations effectué sur les différentes composantes du signal est réalisé par des amplificateurs opérationnels présentant d'excellentes caractéristiques telles que :

- de très faibles courants de polarisation $\left(\simeq 10^{-15} \mathrm{~A}\right)$;

- de très faibles tension d'offset $(\simeq \mu \mathrm{V})$ à faible dérive en température $\left(\simeq 1 \mu \mathrm{V} /{ }^{\circ} \mathrm{C}\right)$;

- de très fortes impédances d'entrée $\left(\simeq 10^{15} \Omega\right)$.

Les composants passifs utilisés sont eux aussi de très bonne qualité. Les différentes parties du montage sont blindées et l'ensemble est placé dans une cage de Faraday dont l'alimentation est filtrée. Les expériences décrites par la suite sont réalisées à une fréquence de $20 \mathrm{~Hz}$.

Une représentation schématique du système électronique est donnée à la figure 3 . A chaque étage du détecteur correspond une opération particulière effectuée sur le signal et décrite dans la partie " principe de la détection».

3.2.2 Analyse électrique du circuit. - Dans un domaine de fréquences, extérieur à celui dans lequel existe une interaction forte entre les dipôles intrinsèques du matériau et le champ électrique appliqué, un diélectrique peut être représenté par un circuit composé d'une résistance en parallèle sur une capacité. Cette représentation va nous permettre d'établir la fonction de transfert de notre détecteur pour des tensions inférieures à $V_{c \text { c. }}$ et d'en faire apparaître la limitation en sensibilité.

De l'analyse de la branche haute tension (échantillon 2, tension $V_{2}$ ), il vient :

$$
I_{2}=\left(\frac{1}{R_{2}}+j C_{2} \omega\right) m A \sin \omega t
$$

avec $I_{2}$ le courant circulant dans l'échantillon 2 de résistance $R_{2}$ et de capacité $C_{2}$ soumis à une tension $V_{2}=m V_{1}=m A \sin \omega t$. 


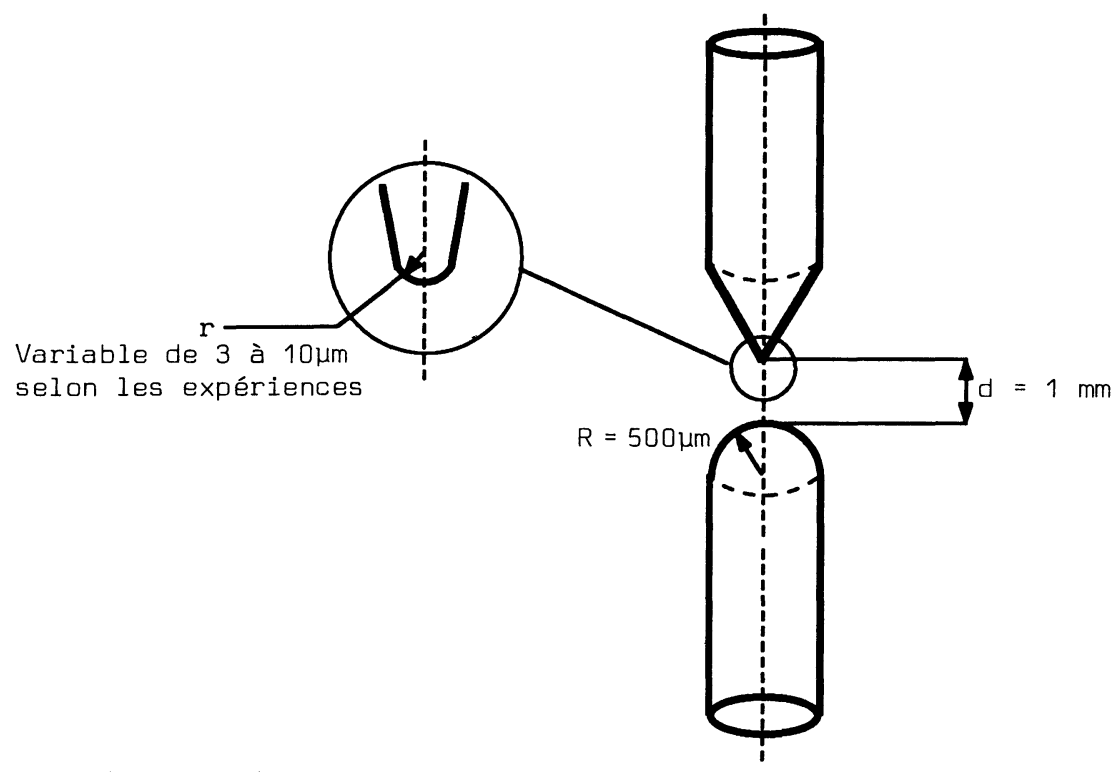

Fig. 2. - Configuration des électrodes.

[Electrode configuration.]

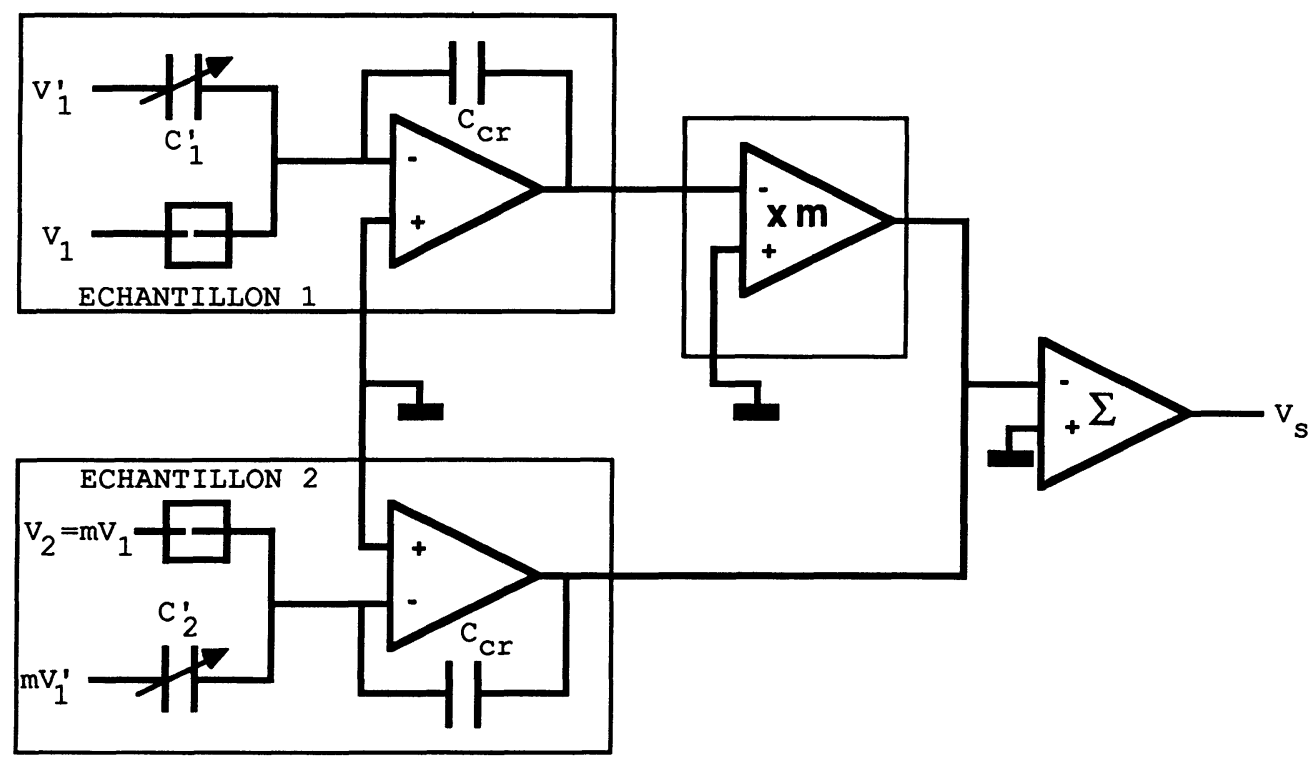

Fig. 3. - Représentation schématique du système électronique de détection de la charge d'espace. $C_{1}^{\prime}$ et $C_{2}^{\prime}$ sont les capacités à air, $C_{\mathrm{cr}}$, la capacité de contre-réaction.

[Schematic representation of the electronic circuit. $C_{1}^{\prime}$ and $C_{2}^{\prime}$ are air capacitors, $C_{\mathrm{cr}}$, the feedback capacitor.]

A $I_{2}$ est ajouté un courant $I_{2}^{\prime}$ provenant de l'application d'une tension en opposition de phase sur un condensateur à air supposé parfait de capacité $C_{2}^{\prime}$ et tel que :

$$
I_{2}^{\prime}=j C_{2}^{\prime} \omega B \sin (\omega t+\pi) .
$$

Le courant total à l'entrée de l'amplificateur opérationnel, côté haute tension, est alors :

$$
\begin{aligned}
I_{\mathrm{t} 2}=I_{2}+I_{2}^{\prime} & = \\
& =\left(\frac{m A}{R_{2}}+j \omega\left(C_{2} m A-B C_{2}^{\prime}\right)\right) \sin \omega t .
\end{aligned}
$$

La compensation de la composante capacitive est réalisée dès que :

$$
m A C_{2}=B C_{2}^{\prime} .
$$

L'analyse de la branche basse tension (échantillon 1 , tension $V_{1}$ ), conduit de manière identique à une expression d'équilibre du type :

$$
A C_{1}=B^{\prime} C_{1}^{\prime}
$$

si $C_{1}$ est la capacité de l'échantillon 1 et $B^{\prime} \sin (\omega t+\pi)$ la tension appliquée à un autre condensateur à air de capacité $C_{1}^{\prime}$. 
A la sortie du convertisseur courant/tension, les signaux équilibrés deviennent respectivement, côté haute tension

$$
V_{\mathrm{s} 2}=-\frac{1}{C_{\mathrm{cr}}} \int_{0}^{t}\left(\frac{m A}{R_{2}}\right) \sin \omega t \mathrm{~d} t
$$

et côté basse tension :

$$
V_{\mathrm{s} 1}=-\frac{1}{C_{\mathrm{cr}}} \int_{0}^{t}\left(\frac{A}{R_{1}}\right) \sin \omega t \mathrm{~d} t .
$$

Si les deux échantillons étaient strictement identiques, on aurait $R_{1}=R_{2}$. En fait, et quel que soit le soin apporté à leur préparation, tel n'est jamais le cas. Afin de minimiser l'erreur introduite dans la mesure, la multiplication théorique du signal par un facteur $m$ au niveau du deuxième étage du détecteur est en réalité effectuée par un facteur $m^{\prime}$, avec:

$$
m^{\prime}=\lim _{R_{1} \rightarrow R_{2}} m .
$$

On obtient donc, à la sortie du deuxième étage et côté basse tension :

$$
V_{\mathrm{sl}}=\frac{1}{\mathrm{C}_{\mathrm{cr}}} \int_{0}^{t}\left(\frac{m^{\prime} A}{R_{1}}\right) \sin \omega t \mathrm{~d} t
$$

compte-tenu du montage inverseur utilisé.

Le dernier étage est un sommateur de gain unité, et le signal de sortie devient :

$$
\begin{aligned}
V_{\mathrm{st}} & =-\left(V_{\mathrm{s} 2}+V_{\mathrm{s} 1}\right) \\
& =\frac{A}{C_{\mathrm{cr}}} \int_{0}^{t}\left(\frac{m}{R_{2}}-\frac{m^{\prime}}{R_{1}}\right) \sin \omega t \mathrm{~d} t .
\end{aligned}
$$

Si l'on pose alors :

$$
R^{\prime}=\frac{R_{1} R_{2}}{m R_{1}-m^{\prime} R_{2}}
$$

la relation (10) devient :

$$
V_{\mathrm{s}}=\frac{1}{C_{\mathrm{cr}}} \int_{0}^{t} \frac{A}{R^{\prime}} \sin \omega t \mathrm{~d} t .
$$

En introduisant la quantité

$$
Q_{\mathrm{r}}=\int_{0}^{t} \frac{A}{R^{\prime}} \sin \omega t \mathrm{~d} t
$$

dite charge résiduelle, l'équation (8) devient :

$$
V_{\mathrm{s}}=\frac{Q_{\mathrm{r}}}{C_{\mathrm{cr}}}
$$

Notons que si les deux échantillons étaient strictement identiques, la charge résiduelle serait nulle. C'est la valeur de cette charge qui limite la sensibilité de notre détecteur. La charge résiduelle varie linéai- rement et en phase avec la tension appliquée, ce qui nous permettra d'observer la charge d'espace $Q_{\mathrm{ce}}$ comme toute déviation à ce comportement linéaire puisque nous avons :

$$
\begin{array}{ll}
V<V_{\mathrm{c}} & V_{\mathrm{s}}=\frac{Q_{\mathrm{r}}}{C_{\mathrm{cr}}} \\
V>V_{\mathrm{c}} & V_{\mathrm{s}}=\frac{\left(Q_{\mathrm{r}}+Q_{\mathrm{ce}}\right)}{C_{\mathrm{cr}}} .
\end{array}
$$

\section{Résultats expérimentaux et discussion.}

L'annulation des composantes capacitive et de polarisation est réalisée à basse tension $\left(m V_{1}=200 \mathrm{~V}\right.$ efficaces), conditions dans lesquelles on peut évidemment négliger les phénomènes d'injection (champ effectif en pointe de l'électrode du côté haute tension $\simeq 16 \mathrm{kV} / \mathrm{mm}$ pour $r=3 \mu \mathrm{m}$ ). Dans les paragraphes suivants, cette opération est dite : " équilibrage du détecteur ".

La tension est ensuite incrémentée par palliers de $200 \mathrm{~V}$ toutes les minutes, ce qui permet la mesure précise de l'arnplitude crête à crête de la charge visualisée sur l'écran d'un oscilloscope à mémoire.

4.1 LA CHARGE RÉSIDUELLE. - Le comportement théorique de la charge résiduelle a tout d'abord été vérifié dans le cas d'une configuration d'électrode non injectante $(r=500 \mu \mathrm{m})$. On observe bien une variation linéaire et en phase du signal de sortie avec la tension appliquée. La figure 4 montre l'évolution de la charge résiduelle $Q_{\mathrm{r}}$ pour différentes valeurs du rayon de courbure $r$, pour des tensions inférieures à la tension critique. On peut ainsi noter que plus $r$ est grand, plus la charge résiduelle est importante.

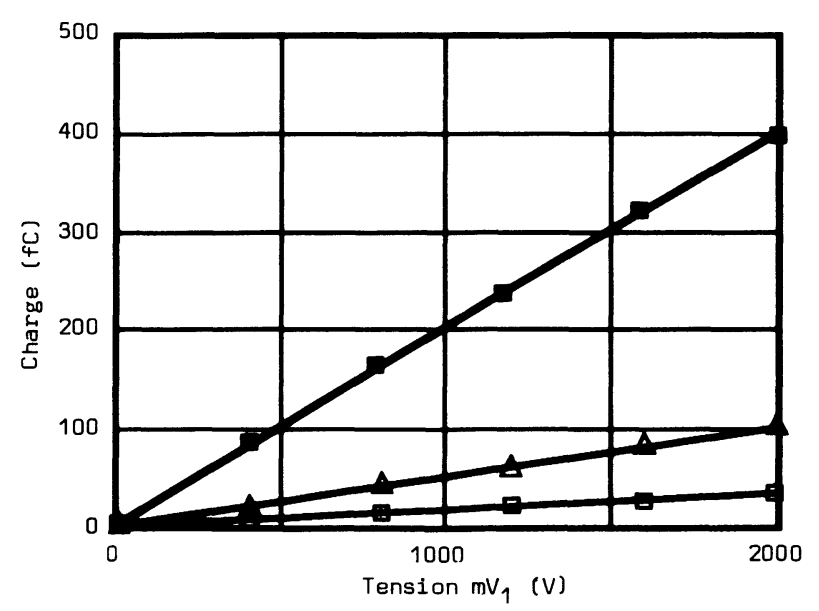

Fig. 4. - Evolution de la charge résiduelle $Q_{\mathrm{r}}$ pour différents rayons de courbure $r$ dans le cas où la tension critique $V_{\mathrm{c}}$ n'est pas atteinte (cas du polyépoxy).

[The residual charge $Q_{\mathrm{r}}$ versus voltage for different curvature radius $r$ and $V<V_{\mathrm{c}}$ (polyepoxy). (四) $r=500 \mu \mathrm{m} ;(\triangle) r=11 \mu \mathrm{m}$; (ㅁ) $r=5 \mu \mathrm{m}$.] 
Cette observation peut être rapprochée des résultats obtenus lors de la mesure de la résistance transversale des matériaux. Dans le cas de nos échantillons, c'est alors la contribution de chaque élément de surface des électrodes à la résistance de l'objet qui dépend du rayon de courbure. Ce comportement de la charge résiduelle a été observé aussi bien sur des échantillons de polyépoxy que sur des échantillons de polyéthylène. La valeur de cette charge est cependant différente, pour une même configuration d'électrode, d'un polymère à l'autre (Fig. 5). C'est donc que leur résistivité n'est pas identique, comme le confirment d'ailleurs des mesures de pertes diélectriques.

Ces expériences montrent à elles seules l'intérêt du blindage de l'électrode active pratiqué par Hibma et al. [7] qui permet une diminution très importante de l'admittance complexe de l'échantillon et conduit par là même à une meilleure sensibilité de la mesurc $\left(Q_{\mathrm{r}} \simeq 0, Q_{\mathrm{ce}} \simeq 10^{-15} \mathrm{C}\right)$.

\subsection{DÉTERMINATION EXPÉRIMENTALE DES CHAMPS} CRITIQUES D'INJECTION. - Nous allons voir qu'il est possible de mesurer $Q_{\mathrm{ce}}$ avec précision, malgré la présence de la charge résiduelle.

Nous avons porté à la figure 6 les variations expérimentales de la charge totale $Q_{\mathrm{t}}=Q_{\mathrm{r}}+Q_{\mathrm{ce}}$ en fonction de la tension appliquée aux échantillons selon le protocole précédemment décrit. Les deux courbes correspondent à deux valeurs du rayon de courbure de l'électrode active. Les résultats présentés sont relatifs à des échantillons de polyépoxy, mais les variations sont qualitativement identiques pour des échantillons de polyéthylène. A faible tension d'alimentation, on note l'évolution linéaire

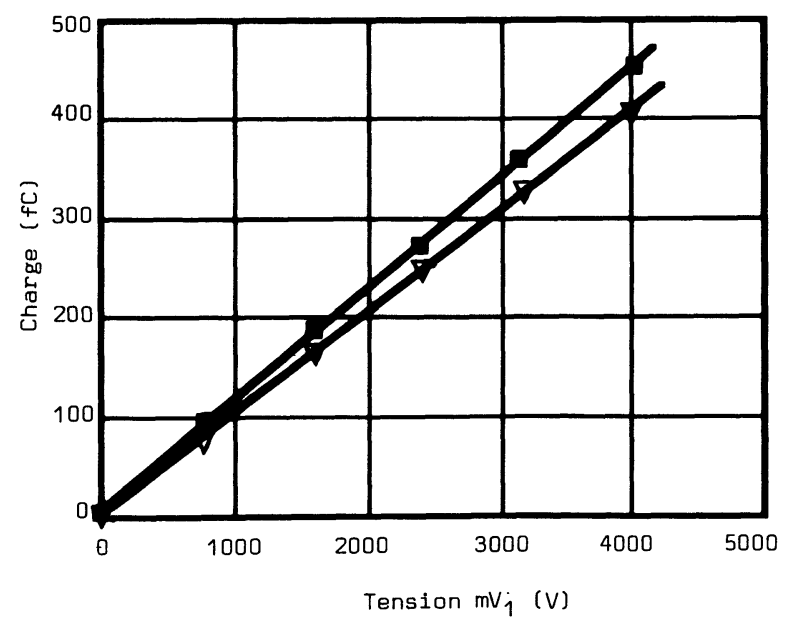

Fig. 5. - Comparaison des charges résiduelles des deux matériaux étudiés, pour $r=40 \mu \mathrm{m}$.

[The residual charge $Q_{\mathrm{r}}$ versus voltage for polyethylene and epoxy samples $(r=40 \mu \mathrm{m})$. ( $\square)$ polyethylene; $(\nabla)$ polyepoxy.]

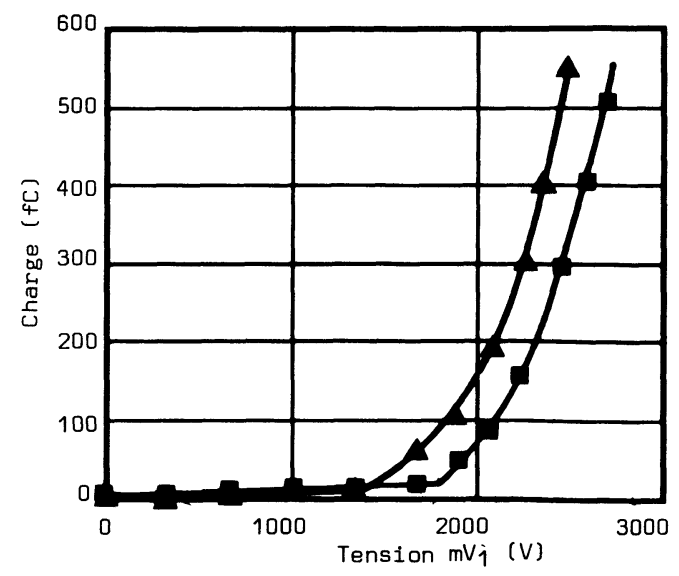

Fig. 6. - Variation expérimentale de la charge totale en fonction de la tension pour deux valeurs du rayon de courbure.

[The total charge versus voltage for two curvature radii

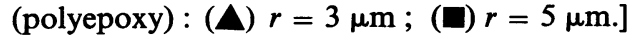

due à la charge résiduelle. Le comportement supralinéaire est observé à partir de la tension critique d'injection qui dépend donc de $r$ puisque nous avons :

$$
\begin{aligned}
& -V_{\mathrm{c}}(r=3 \mu \mathrm{m}) \simeq 1350 \mathrm{~V} \\
& -V_{\mathrm{c}}(r=5 \mu \mathrm{m}) \simeq 1800 \mathrm{~V} .
\end{aligned}
$$

Par application de la relation (3), ces tensions conduisent cependant à une même valeur du champ électrique, qui est donc le champ critique d'injection. Notons que l'invariance du champ critique en fonction de $r$ a déjà été rapportée [7] pour la gamme des rayons de courbure s'étendant de 1 à 10 microns.

Des expériences réalisées sur un grand nombre d'échantillons permettent de déterminer l'écart statistique dû à la dispersion des mesures. On a :

$$
-E_{\mathrm{c}}=0,9 \pm 0,2 \mathrm{MV} / \mathrm{cm} \text {. }
$$

La dispersion est donc de l'ordre de $25 \%$ dans le cas du polyépoxy. Les résultats obtenus dans le cas du polyéthylène conduisent à :

$$
-E_{\mathrm{c}}=1,2 \pm 0,6 \mathrm{MV} / \mathrm{cm}
$$

L'écart statistique est plus important dans ce cas $(\simeq 50 \%$ ).

Nous avons reporté à la figure 7 les variations expérimentales de la charge $Q_{\mathrm{ce}}=\left(Q_{\mathrm{t}}-Q_{\mathrm{r}}\right)$ relatives à des échantillons de polyépoxy et de polyéthylène et obtenues pour $r=3 \mu \mathrm{m}$, en fonction de $\left(V-V_{c}\right)$. On constate que les courbes sont superposables ce qui montre que la quantité de charges mise en jeu dans nos échantillons polymères n'est fonction que de la différence entre la tension critique et la tension appliquée. 


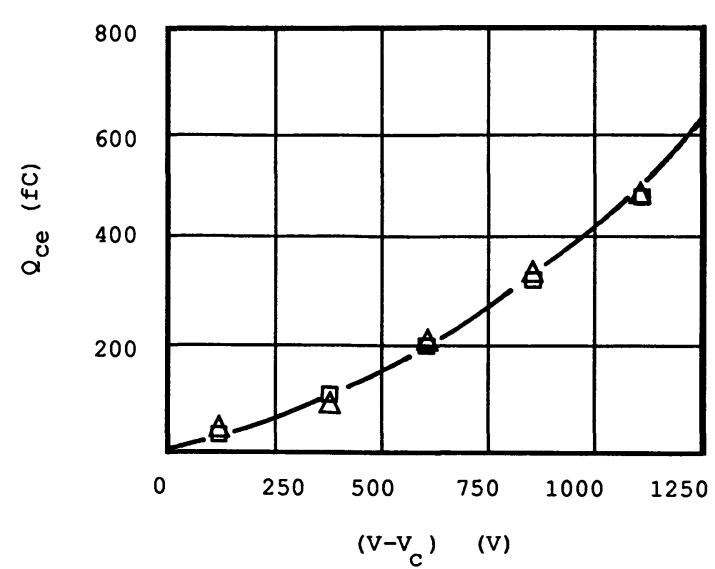

Fig. 7. - Variation expérimentale de la charge $Q_{\mathrm{ce}}=\left(Q_{\mathrm{t}}-Q_{\mathrm{r}}\right)$ en fonction de $\left(V-V_{\mathrm{c}}\right)$ pour les polymères de l'étude.

[Charge injection $Q_{\mathrm{ce}}=\left(Q_{\mathrm{t}}-Q_{\mathrm{r}}\right)$ versus the overcritical voltage $\left(V-V_{\mathrm{c}}\right)(r=3 \mu \mathrm{m}):(\Delta)$ polyethylene; (ㅁ) polyepoxy.]

4.3 INFLUENCE D'UNE POLARISATION PRÉALABLE DES ÉCHANTILLONS. - Intéressons nous au comportement des échantillons lorsque la tension est ramenée, après une première détermination de la valeur du champ critique, à sa valeur d'équilibre soit $V_{2}=m V_{1}=200 \mathrm{~V}$. Dans tous les cas et quel que soit le matériau, les conditions d'équilibre de l'échantillon dont la tension critique a été excédée ne sont plus satisfaites. A l'inverse, l'échantillon 1 qui n'a jamais atteint $V_{\mathrm{c}}$ n'est pas affecté. Si la composante capacitive du signal relatif à l'échantillon 2 est annulée, le signal résistif qui en résulte est environ un ordre de grandeur supérieur au signal résistif relatif à l'équilibrage initial. Ce comportement est décrit à la figure 8 sur laquelle nous avons fait figurer les signaux résistifs correspondants à ces deux situations.

De même, une nouvelle détermination du champ critique se traduit par une diminution de sa valeur (Fig. 9). Ce n'est qu'après une mise en court-circuit

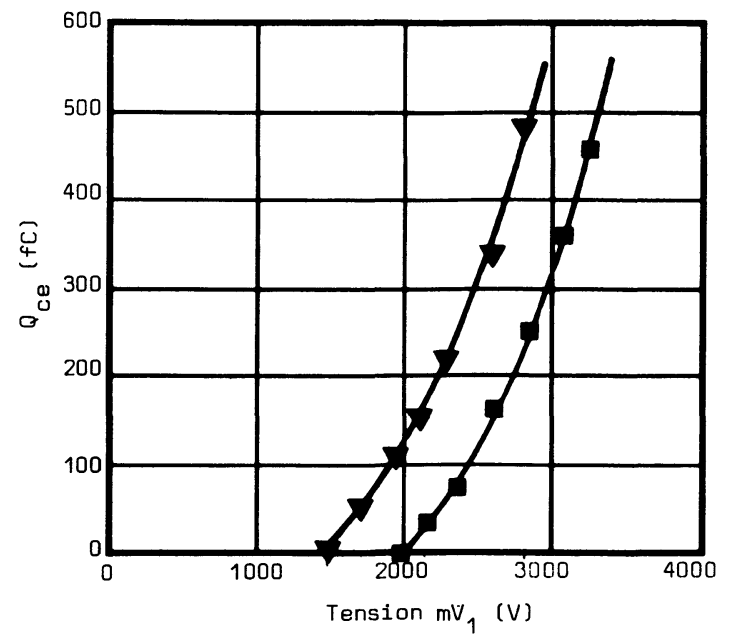

Fig. 9. - Variation de la charge $Q_{\mathrm{ce}}$ en fonction de la tension $(r=6 \mu \mathrm{m})$ (polyepoxy): ( $\square$ ) lors d'une première détermination;

(A) lors d'une deuxième détermination effectuée immédiaement après la première.

[Charge injection $Q_{\mathrm{ce}}$ versus voltage in a polyepoxy sample $(r=6 \mu \mathrm{m})$ : ( $\square)$ first determination of $V_{\mathrm{c}} ;(\Delta)$ second determination of $V_{c}$ realized just after the first one.]

de plusieurs jours, fonction de la quantité de charges préalablement injectée, que la valeur initiale du champ critique est retrouvée.

Ce comportement prouve qu'une charge permanente peut être créée dans un diélectrique sous tension alternative.

\subsection{VISUALISATION DE LA CHARGE INJECTÉE SOUS} TENSION ALTERNATIVE. - La visualisation du signal de sortie du détecteur est importante puisqu'elle donne accès aux variations temporelles de la charge injectée. Dans la pratique, cette observation est limitée par la nature même du signal de sortie $\left(Q_{\mathrm{t}}=Q_{\mathrm{r}}+Q_{\mathrm{ce}}\right)$, et par son évolution dans le temps. C'est la raison pour laquelle nous serons d'abord amené à analyser la signification de la forme du

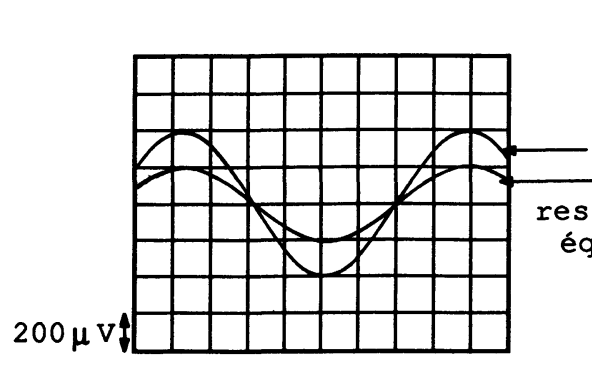

(a)

$$
\mathrm{f}=20 \mathrm{hz}
$$

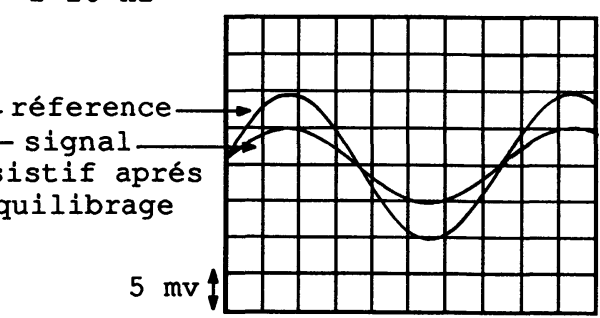

(b)

Fig. 8. - Evolution du signal résistif mesuré à faible tension d'alimentation $\left(m V_{1}=200 \mathrm{~V}\right)$ aux bornes de l'échantillon 2 (polyéthylène) : a) avant détermination de $V_{\mathrm{c}} ;$ b) après détermination de $V_{\mathrm{c}}$.

[The inphase component of the charge at sample's 2 terminal at low input voltage $\left(m V_{1}=200 \mathrm{~V}\right)$ (polyethylene). a) before $V_{\mathrm{c}}$ determination ; b) after $V_{\mathrm{c}}$ determination.] 
signal de sortie observée juste après l'équilibrage du détecteur (court terme), puis en fonction du temps (long terme).

4.4.1 Signification du signal de sortie pour une observation à court terme : visualisation directe de la charge injectée. - Dès que la tension critique est atteinte, le signal de sortie est la composition de deux signaux, $Q_{\mathrm{r}}+Q_{\mathrm{ce}}$. La charge d'espace ne sera donc observée dans des conditions satisfaisantes que pour $Q_{\mathrm{r}} \ll Q_{\mathrm{ce}}$. L'évolution de la forme du signal de sortie en fonction de la tension appliquée est représentée à la figure 10. Pour $V>V_{\mathrm{c}}$, avec $V / V_{\mathrm{c}} \simeq 1$, on a $Q_{\mathrm{r}} \simeq Q_{\mathrm{ce}}$. La forme du signal de sortie (cf. Fig. 10) résulte de la composition de deux signaux du même ordre de grandeur, mais légèrement déphasés l'un par rapport à l'autre. Même si l'on peut en déduire que la charge injectée ne varie pas en phase avec la tension appliquée aux échantillons, la visualisation du signal ne présente que peu d'intérêt dans ces conditions. Par contre, pour $V / V_{\mathrm{c}} \simeq 2$, on a $Q_{\mathrm{ce}} / Q_{\mathrm{r}} \simeq 20$. On peut alors négliger la contribution de la charge résiduelle au signal total et considérer sa

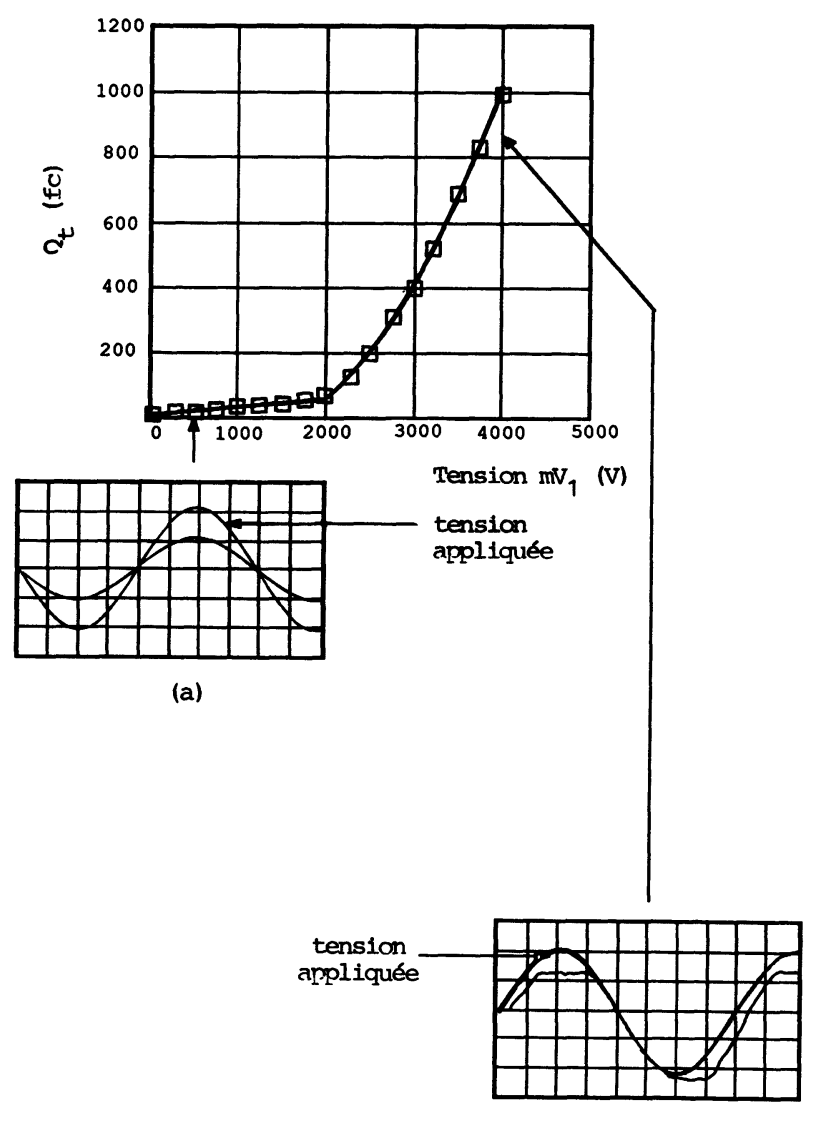

(b)

Fig. 10. - Visualisation de la charge totale pour différents niveaux de tension (polyepoxy).

[The total charge vizualisation at different voltage levels (polyepoxy): a) $V<V_{\mathrm{c}} ; \quad Q_{\mathrm{t}}=Q_{\mathrm{r}} ; \quad$ b) $V>V_{\mathrm{c}}$; $Q_{\mathrm{ce}} \gg Q_{\mathrm{r}} ; Q_{\mathrm{t}} \simeq Q_{\mathrm{ce}}$.] forme comme représentative des variations temporelles de la charge injectée. On en déduit que l'injection de charge est ambipolaire, qu'elle s'effectue durant l'augmentation de la tension excitatrice mais que le courant d'injection $\mathrm{d} Q / \mathrm{d} t$ s'annule avant les maximums de tension. Ce phénomène de saturation, traduit par les parties quasi-plates du signal, résulte des modifications du champ électrique local dues à la charge d'espace. Un calcul simple montre d'ailleurs que la quantité de charge mise en jeu au cours d'une alternance $\left(\simeq 0,5 \times 10^{-12} \mathrm{C}\right.$ pour $V / V_{\mathrm{c}}=2$ d'après nos expériences), réparties sur une distance de quelques microns $(\simeq 2 \mu \mathrm{m}$ pour $V \simeq 2 V_{c}$, [7]), modifie considérablement le champ en pointe qui n'est donc plus calculable par la relation (3). La quantité de charge injectée durant les alternances négatives est supérieure à celle mise en jeu durant les alternances positives. Ce comportement assymétrique de la charge injectée vis-à-vis du diélectrique avait déjà été rapporté dans le cas du polyéthylène [1]. Cet effet de polarité est observé de la même façon dans le polyépoxy de l'étude.

La non simultanéité du passage à zéro des deux signaux est intéressante à étudier puisqu'elle permet un certain nombre de déduction quant au dépiégeage des porteurs injectés. Au passage à zéro lorsque la tension devient positive, le signal de sortie est en retard de quelques ms sur la tension : c'est donc que des électrons restent dans le diélectrique alors que la tension appliquée est déjà positive. Ce phénomène n'est pas observé dans le cas des trous (charges positives) puisqu'il y a simultanéité du passage à zéro des deux signaux lorsque la tension devient négative. On en déduit que les électrons sont plus difficilement extraits du diélectrique que les trous. Les électrons seraient alors, soit plus profondément piégés que les trous, soit piégés plus loin de l'électrode compte-tenu de leurs mobilités plus importantes.

4.4.2 Signification du signal de sortie pour une observation à long terme. - L'enregistrement du signal de sortie en fonction du temps montre que ses caractéristiques (forme, amplitude et angle de phase) évoluent rapidement. Après un temps $t_{0}$, le signal devient purement sinusoïdal, d'amplitude $Q_{0}$ et d'angle de phase $\phi_{0}$ (Fig. 11). Les valeurs $t_{0}$, $Q_{0}$ et $\phi_{0}$ dépendent principalement du rayon de courbure $r$ de l'électrode active et du rapport $V / V_{\text {c }}$. L'évolution de $Q$ et $\phi$ est donnée en fonction du temps à la figure 12 dans le cas particulier où $r=3 \mu \mathrm{m}$ et $V / V_{\mathrm{c}}=1,4$. Les valeurs $t_{0}, Q_{0}$ et $\phi_{0}$ ont été portées au tableau I en fonction du rayon de courbure, pour le même rapport $V / V_{\mathrm{c}} . \phi_{0}$ et $Q_{0}$ sont plus grands dans le cas d'un rayon $r=5 \mu \mathrm{m}$ tandis que $t_{0}$ est plus faible.

Notons que dans une configuration non injectante, le signal de sortie, dans ce cas uniquement dû à la 
a)

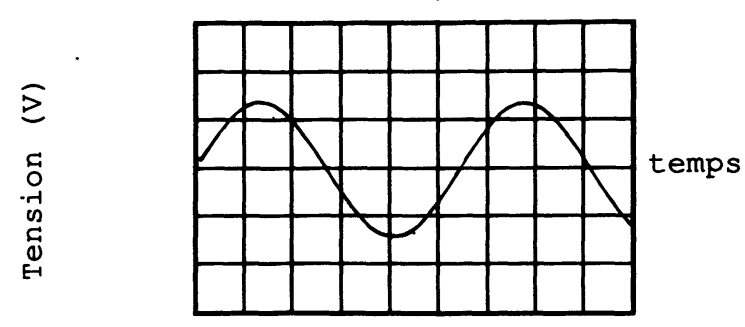

b)

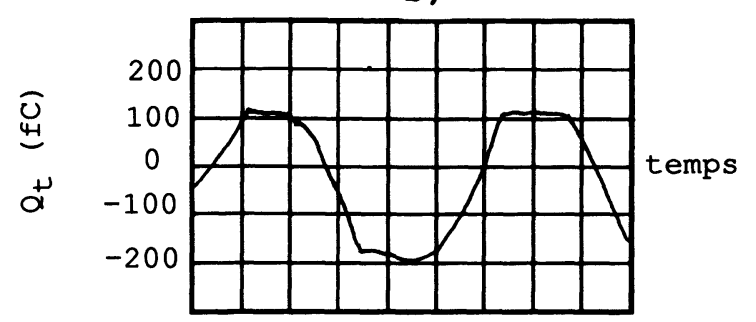

c)

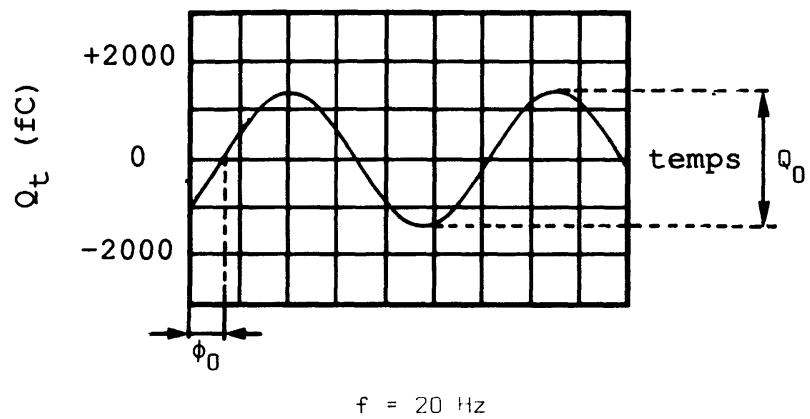

Fig. 11. - Evolution temporelle de la forme du signal de sortie (charge totale) dans le cas d'une configuration injectante (polyepoxy) : a) tension appliquée; b) signal initial $\left.(t) ; V / V_{\mathrm{c}}=1,4 ; \mathrm{c}\right)$ signal final $t_{0}=t+60 \mathrm{~min}$.

[Temporal evolution of the signal wave form for an injecting needle configuration (polyepoxy): a) applied voltage ; b) just after the bridge tuning $(t) ; V / V_{\mathrm{c}}=1.4$; c) one hout later.]

charge résiduelle, ne change pas dans la limite de la durée de nos observations (70 heures). L'évolution rapportée dans le cas d'une configuration injectante n'est donc pas liée à une dérive de l'électronique du détecteur. Notons enfin que ce comportement sinusoïdal a été observé par Fruth et al. [10] dans des conditions similaires.

Compte-tenu de ces éléments, on peut conclure que l'évolution décrite est liée à une modification des conditions de conduction dans le diélectrique. Une nouvelle contribution au signal de sortie, non prise en compte au niveau de la simulation électrique des échantillons, vient donc s'ajouter au terme $\left(Q_{\mathrm{r}}+Q_{\mathrm{ce}}\right)$ pour les dominer rapidement. Tout se passe en fait comme si une partie de la charge injectée restait piégée durablement dans le matériau. Compte-tenu du comportement non symétrique du diélectrique vis-à-vis des porteurs de charge (cf.
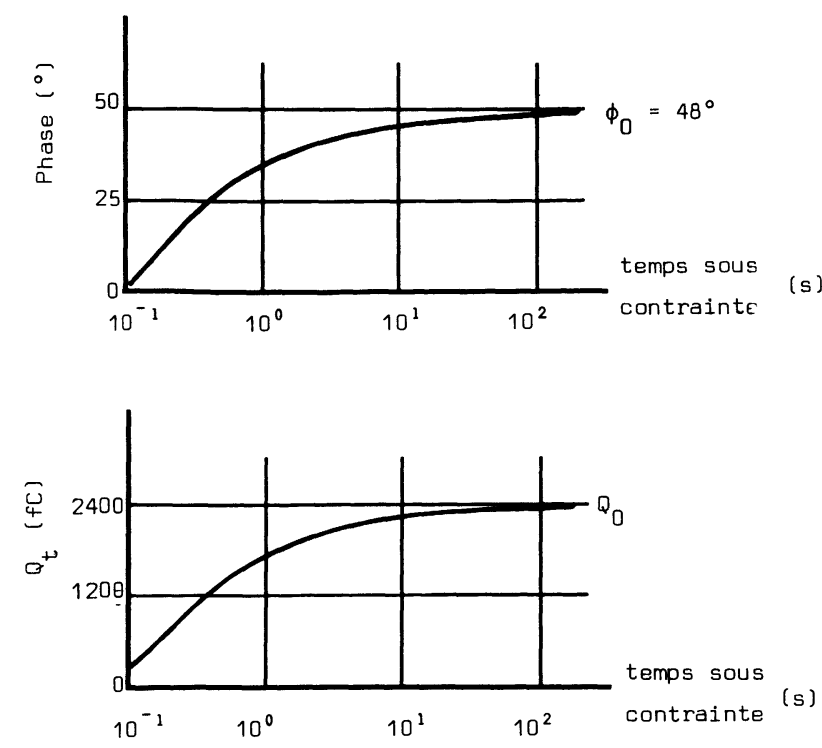

Fig. 12. - Evolution temporelle de l'amplitude $Q$ de la charge de sortie et de sa phase. $r=3 \mu \mathrm{m} ; V / V_{\mathrm{c}}=1,4$; (epoxy).

[Temporal evolution of the output signal phase and amplitude $\left(r=3 \mu \mathrm{m}, V / V_{\mathrm{c}}=1.4\right.$, epoxy).]

Tableau I. - Valeur de $t_{0}, Q_{0}, \phi_{0}$ en fonction $d u$ rayon de courbure pour un même rapport $V / V_{\mathrm{c}}$ (polyépoxy).

$\left[t_{0}, Q_{0}, \phi_{0}\right.$, values versus curvature radius $r$ for $V / V_{\mathrm{c}}=1.4$ in polyepoxy samples.]

\begin{tabular}{|c|c|c|c|}
\hline $\begin{array}{c}r \\
(\mu \mathrm{m})\end{array}$ & $\begin{array}{c}Q_{0} \\
(\mathrm{fC})\end{array}$ & $\begin{array}{c}\phi_{0} \\
\left(^{\circ}\right)\end{array}$ & $\begin{array}{c}t_{0} \\
(\mathrm{~min})\end{array}$ \\
\hline 3 & 2000 & 48 & $\simeq 60$ \\
\hline 5 & 2800 & 60 & $\simeq 50$ \\
\hline
\end{tabular}

paragraphe précédent), cette charge permanente serait plutôt négative. Soit $Q_{\mathrm{cp}}$ cette charge. Le signal de sortie du détecteur s'écrit maintenant :

$$
Q_{\mathrm{t}}=Q_{\mathrm{r}}+Q_{\mathrm{ce}}+Q_{\mathrm{cp}} \text {. }
$$

Il est d'ailleurs possible de compenser les composantes capacitives et polaires de cette nouvelle charge en cours d'expérimentation. Le signal observé après cette compensation présente les mêmes caractéristiques que $Q_{\text {ce }}$ ce qui prouve qur les phénomènes en pointe ne sont pas qualitativement différents de ceux observés quand $Q_{\mathrm{cp}}=0$. La situation à la pointe de l'électrode active serait alors celle décrite à la figure 13. La quantité de charge durablement piégée dans le diélectrique augmente avec le temps et devient la contribution majeure au signal de sortie. La différence notée entre $\phi_{0}, Q_{0}$ et $t_{0}$ en fonction du rayon de courbure $r$ pourrait être interprétée en 

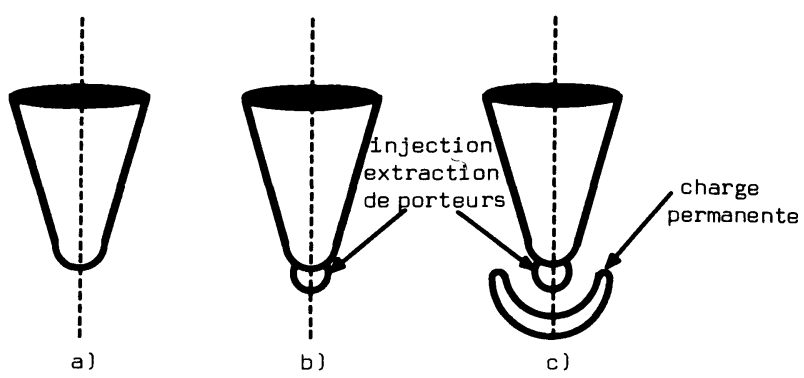

Fig. 13. - Evolution schématique de la charge en pointe : a) pas d'injection; b) avec injection, observation à court terme ; c) avec injection, observation à long terme.

[Schematic situation at needle tip: a) with no space charge injection; b) with space charge injection, short term ; c) with space charge injection, long term.]

remarquant que la quantité de charge injectée dans la diélectrique est d'autant plus importante que $r$ est grand, à rapport constant $V / V_{\mathrm{c}}$. Les données expérimentales (Fig. 6) conduisent en effet à :

$$
\begin{aligned}
& Q_{\mathrm{ce}} \simeq 100 \times 10^{-15} \mathrm{C} \text { pour } r=3 \mu \mathrm{m} \\
& Q_{\mathrm{ce}} \simeq 300 \times 10^{-15} \mathrm{C} \text { pour } r=5 \mu \mathrm{m}
\end{aligned}
$$

pour $V / V_{\mathrm{c}}=1,4$.

La quantité de charge injectée $Q_{c e}$, et par conséquent la charge permanente $Q_{\mathrm{cp}}$ est plus importante dans le cas où $r=5 \mu \mathrm{m}$ ce qui conduit d'une part à des valeurs $\phi_{0}$ et $Q_{0}$ plus importantes, et d'autre part à une faible valeur de $t_{0}$.

La simulation de l'évolution du signal de sortle nécessite la connaissance de $Q_{\mathrm{cp}}(t)$. Une approche du problème a été tentée en utilisant le modèle de Maxwell-Wagner à deux couches dans lequel l'échantillon est maintenant représenté par deux diélectriques en série simulant les régions du matériau avec et sans charge permanente. L'évolution expérimentale du signal de sortie peut être retrouvée mais une telle simulation analogique n'a que peu d'intérêt dans la mesure où elle ne prend pas en compte les phénomènes physiques.

\section{Conclusion et perspectives : application au vieillis- sement des matériaux.}

Le détecteur de charge mis au point permet l'étude des phénomènes d'injection dans un diélectrique soumis à une contrainte alternative. Sa sensibilité actuelle, principalement limité par l'existence de la charge résiduelle $Q_{\mathrm{r}}$, est de l'ordre de $10^{-14} \mathrm{C}$. Deux grandeurs essentielles sont accessibles: le champ critique d'injection $E_{\mathrm{c}}$ au-delà duquel des porteurs sont injectés massivement dans l'isolant, et la quantité de charge injectée $Q_{\mathrm{ce}}$, ainsi que ses variations temporelles. Dans le cas des matériaux étudiés, un polyéthylène et un polyépoxy, le champ critique est de l'ordre de $1 \mathrm{MV} / \mathrm{cm}$. Sa détermination expérimentale souffre quelquefois d'un écart statistique important vraisemblablement lié à la reproductibilité des échantillons. La charge injectée $Q_{c e}$ peut être visualisée en début d'expérience, mais le signal de sortie du détecteur est rapidement dominé par une nouvelle contribution liée à l'établissement d'une charge permanente $Q_{\mathrm{cp}}$. La quantité de charge $Q_{\mathrm{ce}}$ injectée au cours d'une alternance dépend du détail des conditions expérimentales, mais elle est de l'ordre de $200 \mathrm{fC}$ pour une tension appliquée de $1,5 V_{\mathrm{c}}$. On en déduit qu'au bout d'une heure d'essai, bien avant la rupture diélectrique par arborescence, une quantité de charge de l'ordre de $4000 \mathrm{pC}$ a transité dans le diélectrique. Une caractérisation effectuée après essai ne conduirait qu'à l'évaluation de $Q_{\mathrm{cp}}$ qui n'a que peu de rapport avec la charge réellement mise en jeu. On voit donc tout l'intérêt de la détection sous contrainte.

L'apport du détecteur dans le domaine du vieillissement des isolants est évidente. La détermination expérimentale des tensions critiques, pour une association de matériaux donnée, doit permettre l'évaluation de la résistance au vieillissement d'une interface particulière. C'est en effet au delà de la tension critique d'injection que les porteurs peuvent conduire à des processus dissipatifs potentiellement nocifs pour la tenue du matériau. L'étude de la quantité de charge cumulée mise en jeu jusqu'à la rupture des échantillons, sous niveau de tension donnée, pourrait conduire à des relations d'invariance $(Q, t)$ permettant la prévision des durée de vie d'éprouvettes à partir d'essais à court terme.

\section{Remerciements.}

Les auteurs tiennent à remercier le Société SODERN pour son aide financière et technique.

\section{Bibliographie}

[1] IEDA M., « Electrical conduction and carrier traps in polymeric materials ", Proc. first Int. Conf. on Cond. and Break; in Sol. Diel. (ICSD, Toulouse) juillet 1983, p. 1 .
[2] Lebey T., LAURent C., "Charge injection and electroluminescence as a prelude to dielectric breakdown ", J. Appl. Phys., to be published in 1990. 
[3] VAN TURnHOUT J., "Thermally stimulated discharges of polymers electrets ", (Elsevier, Amsterdam) 1975, p. 109.

[4] Alquié C., Dreyfus J., Lewiner J., "Stress-wave probing of electric field distributions in dielectrics ", Phys. Rev. Lett. 47 (1981) 1483.

[5] Toureille A., "Sur une méthode de détermination de densité spatiale de charge d'espace dans le polyéthylène ", Proc. Sec. Int. Conf. on Poly. Insul. Cable (JICABLE, Paris) Septembre 1987, p. 98.

[6] LEMKE E., « Ein beitrag zur messung impulsloser teitentladungen bei wechselspannung », Elektrie 30 (1976) 479.

[7] Hibma T., Zeller H. R., « Direct measurement of space charge injection from a needle electrode into dielectrics ", J. Appl. Phys. 59 (1986) 1614.

[8] Baumann T., Pfluger P., Strucki F., Zeller H. R., "Charge injection and distribution in polymeric insulation systems ", Proc. of Conf. on Elec. Ins. and Diel. Phen. (CEIDP, New York) octobre 1986, p. 358.

[9] Grinc J., Adamec V., Calderwood J. H., " On the comparability of single and double needle tests for treeing resistance ", IEEE Trans. on Elec. Ins. EI-17, $\mathrm{n}^{\circ} 4$, (1982) p. 356.

[10] Fruth B., BaumanN T., Stucki F., "Space charge injection and partial discharge inception at the metal polymer interface ", Proc. of Third Int. Conf. on Cond. and Break. in Sol. Diel. (ICSD, Trondheim) juillet 1989, p. 35 . 